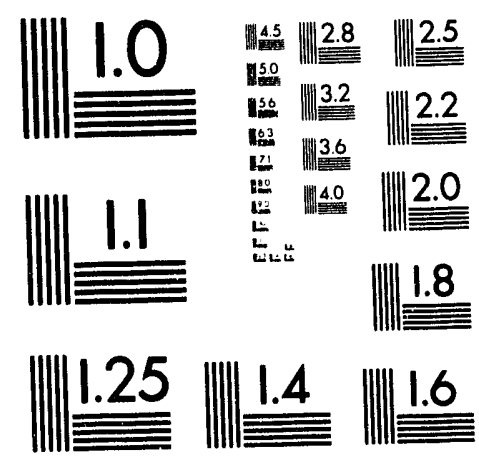



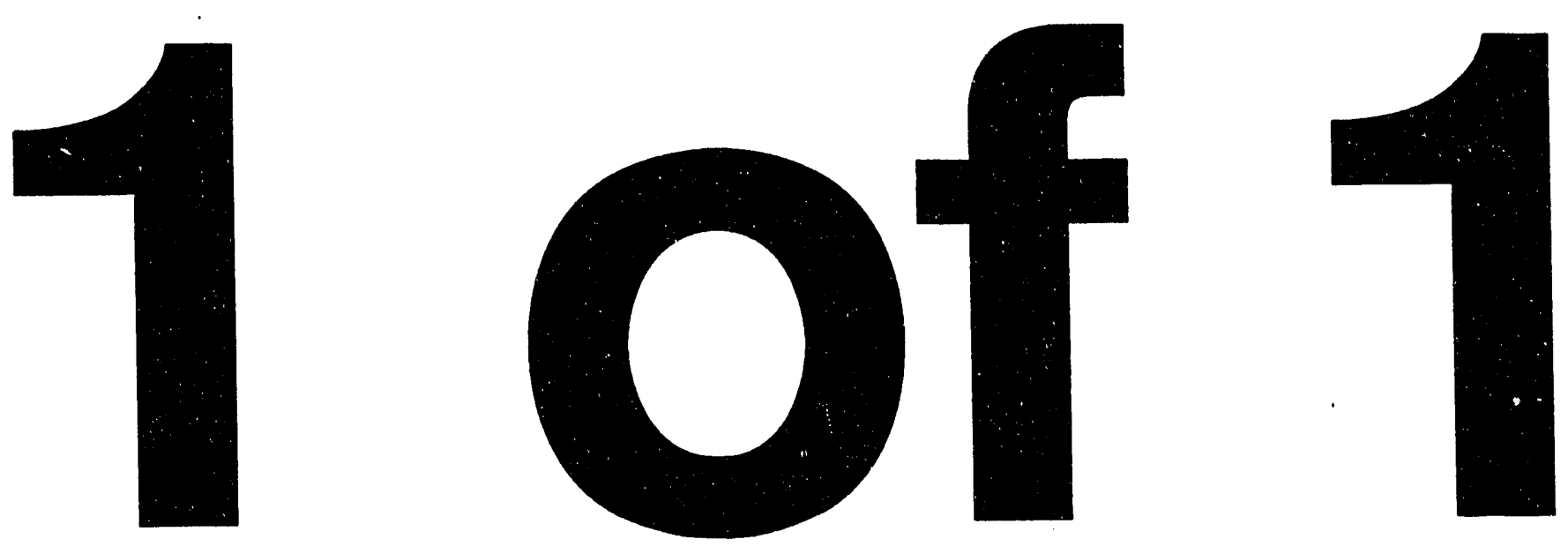
LBL-33270

\title{
Study of Coupled-Bunch Collective Effects in the ALS
}

\author{
J.M. Byrd and J.N. Corlett \\ Accelerator and Fusion Research Division \\ Lawrence Berkeley Laboratory \\ University of California \\ Berkeley, CA 94720
}

May 1993

This work was supported by the Director, Office of Energy Research, Office of Basic Energy Sciences, Materials Sciences Division, of the U.S. Department of Energy under Contract No. DE-AC03-76SF00098.

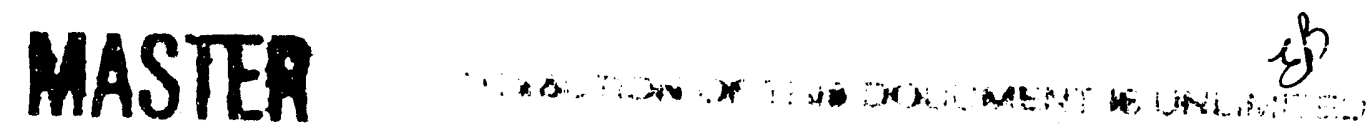




\title{
Study of Coupled-bunch Collective Effects in the ALS*
}

\author{
J. M. Byrd and J. N. Corlett \\ Lawrence Berkeley Laboratory \\ Berkeley, CA 94720 USA
}

\section{Abstract}

We present an overview of the calculated longitudinal and transverse coupled-bunch (CB) growth rates using the measured RF cavity higher order mode (HOM) impedance for the Advanced Light Source (ALS), a $1.5 \mathrm{GeV}$ electron storage ring for producing synchrotron radiation. We also describe a visual method of representing the effective beam impedance and corresponding growth rates which is especially useful for understanding the dependence of growth rate on $H O M$ frequency and $Q$, for determining the requirements of the $\mathrm{CB}$ feedback system, and for interpreting measured beam spectra.

\section{INTRODUCTION}

Because of the high beam current and large number of bunches at the ALS, CB instabilities are predicted to be one of the limiting factors in achieving the design beam intensity while maintaining good beam quality. Longitudinal and transverse CB feedback systems are currently being designed to combat this problem.

\section{COUPLED-BUNCH IMPEDANCE}

In the case of longitudinal beam oscillations, the RF cavities provide the only sources of impedance strong enough to drive $\mathrm{CB}$ instabilities. A series of measurements characterizing the resonant modes of the ALS RF cavity have been performed. A summary of the measurements is given in reference [3], in these proceedings.

In the case of betatron oscillations, there are two sources of impedance large enough to drive $\mathrm{CB}$ instabilities: the RF cavity dipole HOMs and the resistive wall (RW) impedance of the vacuum chamber. A careful estimate of the RW impedance has been made[2], taking into account the variable resistivity, aperture, and cross section of the vacuum chamber around the ring. In addition, the transverse beta functions vary around the ring. Because of the narrower vertical aperture in the chamber, the vertical impedance dominates the horizontal. The net vertical RW impedance, scaled by the ratio of the beta function at the RW impedances and the RF cavities, is given by $Z_{\perp, R W}=0.59 \mathrm{k} \Omega / \mathrm{m} / \sqrt{f(H z)}$.

\section{Coupled-Bunch Growth Rates}

\section{Longitudinal Effects}

The longitudinal complex fiequency shift of $\mathrm{CB}$ mode $\ell$ for a bunched beam with $N$ equally populated and equally

* This work was supported by the Director, Office of Energy Research, Office of Basic Energy Sciences, Materials Sciences Division, of the U.S. Department of Energy under Contract No. DE-AC0376SFOOO98. spaced bunches is given by[4]

$$
\Delta \omega_{\|}^{\ell}=\frac{1}{2} \frac{I_{0} f_{r f} \alpha}{(E / e) Q_{s}} i\left[Z_{\|}\right]_{e f f}^{\ell}
$$

where

$$
\left[Z_{\|}\right]_{e f f}^{l}=\sum_{p=-\infty}^{p=+\infty} \frac{\omega_{p}}{\omega_{r f}} e^{-\left(\omega_{p} \sigma_{r}\right)^{2}} Z_{\|}\left(\omega_{p}\right)
$$

and

$$
\omega_{p}=\left(p N+\ell+Q_{s}\right) \omega_{0} \text {. }
$$

The effective impedance, $\left[Z_{\|}\right]_{e f f}^{\ell}$, represents the sum of the beam spectrum over the actual impedance. The complex frequency shift was evaluated using the measured parameters shown in Table 1 and the measured HOM parameters given in [3]. The imaginary part of $\mathrm{Eq} .1$ represents a growth or damping of the oscillation and is driven by the real part of the impedance. The growth rates were calculated for the worst case of no spread in HOM frequencies between the two RF cells.

Because of the sampling of the cavity wakefields by the beam, the HOM impedances are aliased into a frequency band of $0-1 / 2 T_{b}$, where $T_{b}$ is the bunch separation in time. For nominal ALS conditions, the frequency range is $0-250$ $\mathrm{MHz}$. The summation for the effective impedance given in Eq. 2 represents this aliasing. The real part of the effective longitudinal impedance driving the longitudinal coupled bunch modes is shown in Figure 1. The effective impedance is plotted on the left axis and the growth rates of the unstable beam modes larger than radiation damping are plotted on the right axis. The unstable CB modes themselves are represented by solid vertical lines. The dotted line represents the effective impedance resulting in growth for $\mathrm{CB}$ modes corresponding to upper sidebands in the frequency range $0-250 \mathrm{MHz}$. The dashed line is the effective impedance resulting in growth for lower sidebands in the same frequency range. Note that the dashed line represents a damping impedance for the upper sideband modes in the same frequency range. Several of the aliased HOM's are labeled according to their resonant frequency as given in [3]. The radiation damping rate is also shown in the figure.

The plot of the effective impedance clearly shows the dependence of growth rate on HOM parameters such as resonant frequency, $Q$, and synchrotron tune. For example, if a high- $Q$ HOM is damped, it's width will increase and the peak value of the shunt impedance will decrease. However, as it's width increases, it will drive unstable more $\mathrm{CB}$ modes. In addition, the dependence of growth rate on variations of the HOM frequency resulting from cavity tuner position and water temperature or on changes in 


\begin{tabular}{clc}
\hline \hline Parameter & Description & Value \\
\hline$E_{b}$ & Beam energy & $1.5 \mathrm{GeV}$ \\
$C$ & Circumference & $196.8 \mathrm{~m}$ \\
$f_{r f}$ & RF Freq. & $499.654 \mathrm{MHz}$ \\
$\sigma_{\epsilon}$ & RMS $\delta E / E$ & $7.1 \mathrm{e}-4$ \\
$N$ & Number of bunches & 328 \\
$I_{0}$ & Total DC beam current & $0.4 \mathrm{~A}$ \\
$\alpha$ & momentum compaction & $1.594 \mathrm{e}-3$ \\
$Q_{s}$ & Synchrotron tune & 0.009105 \\
$\sigma_{\ell}$ & RMS bunch length & $3.9 \mathrm{~mm}$ \\
$Q_{x, y}$ & Horizontal betatron tune & $14.28,8.18$ \\
$\beta_{x, y, c a v}$ & $\beta_{x, y}$ at cavity & $11.5 \mathrm{~m}, 5 \mathrm{~m}$ \\
\hline \hline
\end{tabular}

Table 1: ALS parameters used for calculations.

the synchrotron tune is easily found. The plot of the effective impedance is also very useful in interpreting measured spectra of $\mathrm{CB}$ oscillations because of the diurect correlation between CB sidebands and HOMs.

Landau damping from the tune spread due to the nonlinearity of the RF voltage is not effective in damping any of the fast-growing CB modes at full current because of - the short bunch length.

\section{Longitudinal Feedback Threshold}

A CB feedback system is being designed to damp the unstable modes plotted in Fig. 1. Neglecting any aid from radiation damping, the damping limit of the $\mathrm{FB}$ system is reached when the beam-induced voltage/turn equals the maximum FB kick voltage/turn. The magnitude of the beam-induced voltage/turn is given by

$$
V_{b}=I_{0} \Delta \phi Z_{\|}=I_{0} \Delta \phi_{0} Z_{\|, e f f}
$$

where $Z_{\|, e j^{\prime} j}(\omega)=\left(\omega / \omega_{r j}\right) Z_{\|}(\omega)$ is the where $\Delta \phi_{0}$ is the amplitude of phase modulation at the RF frequency for a CB mode. The maximum longitudinal kick/turn is 1.5 $\mathrm{kV}$ and a maximum longitudinal $\mathrm{CB}$ mode amplitude of $0.03 \mathrm{rad}$ is assumed. For ALS injection conditions, all longitudinal $C B$ modes are excited much less than 0.03 rad. The limit of impedance the longitudinal FB can damp is included in Fig. 1.

\section{Transverse Effects}

The transverse complex frequency shift of CB mode $\ell$ for a bunched beam with equally populated and spaced bunches is given by[4]

$$
\Delta \omega_{\perp}^{\ell}=-\frac{1}{2} \frac{I_{0} f_{0}}{(E / e)} i\left[\beta_{\perp} Z_{\perp}\right]_{e \rho f}^{\ell}
$$

where

$$
\left[\beta_{\perp} Z_{\perp}\right]_{e f f}^{\ell}=\beta_{\perp} \sum_{p=-\infty}^{p=+\infty} e^{-\left(\omega_{p} \sigma_{r}\right)^{2}} Z_{\perp}\left(\omega_{p}\right)
$$

and

$$
\omega_{p}=\left(p N+\ell+Q_{\perp}\right) \omega_{0} .
$$

$I_{0}$ is the DC current and $\beta_{\perp}$ is the transverse beta function at the location of the impedance. Using the dipole cavity modes from [3], the complex frequency shift was evaluated the parameters shown in Table 1.

The real part of the effective vertical impedance is shown in Figure 2. The dotted line represents the effective impedance resulting in growth for $C B$ modes corresponding to upper sidebands in the frequency range 0-25i0 $\mathrm{MHz}$. The dashed line is the effective impedance resulting in growth for lower sidebands in the same frequency range.

Landau damping from the intrabunch tune spread due to the nonlinearity of the transverse restoring force is not easy to evaluate. For the assumed value of the tune spread of 0.01 , Landau damping is not effective in damping any of the $\mathrm{CB}$ modes at full beam current although it may help at lower currents. Head-tail damping for the expected strength of the broadband impedance is not significant.

\section{Transverse Feedback Threshold}

Neglecting any aid from radiation damping, the limit of the transverse FB system is reached when the beam-induced voltage/turn equals the maximum FB kick voltage/turn. The magnitude of the beam-induced voltage/turn is given by

$$
V_{b}=I_{0} \Delta x Z_{\perp}
$$

where $\Delta x$ is the transverse oscillation amplitude for a CB mode. The maximum transverse kick/turn is $1.6 \mathrm{kV}$ and a maximum transverse CB amplitude of $1 \mathrm{~mm}$ is assumed. As in the longitudinal case, the transverse FB gain can be raised to sufficiently stabilize a quiescent beam. For ALS injection conditions, all transverse $C B$ modes are excited much less than $1 \mathrm{~mm}$. The limit of the transverse FB is shown in Fig. 2.

\section{CONCLUSIONS}

Longitudinal CB growth rates in the ALS are $\sim 1 / \mathrm{mset}$. Transverse $\mathrm{CB}$ growth rates are $0.5-1 / \mathrm{msec}$. The growth rates are sensitive to detailed RF cavity conditions and betatron tunes. The proposed CB FB systems can damp all unstable modes for the expected injection conditions.

The author would like thank Glen Lambertson for many useful discussions and members of the Center for Beam Physics at LBL for their general support and encouragement.

\section{REFERENCES}

[1] 1-2 GeV Synchrotron Radiation Source-CDR, LBL PUB-5172 Rev., July 1986.

[2] J. Byrd, Resistive Wall Instability at the ALS, ESG Tech Note-195, LSAP-122, May 1992.

[3] J. Corlett, J. Byrd, Measurement of Higher-order Modes of the ALS $500 \mathrm{MHz}$ Accelerating Cavities, these proceedings.

[4] M. Zisman, S. Chattopadhyay, J. Bisognano, ZAP User's manual, LEL-21270. 


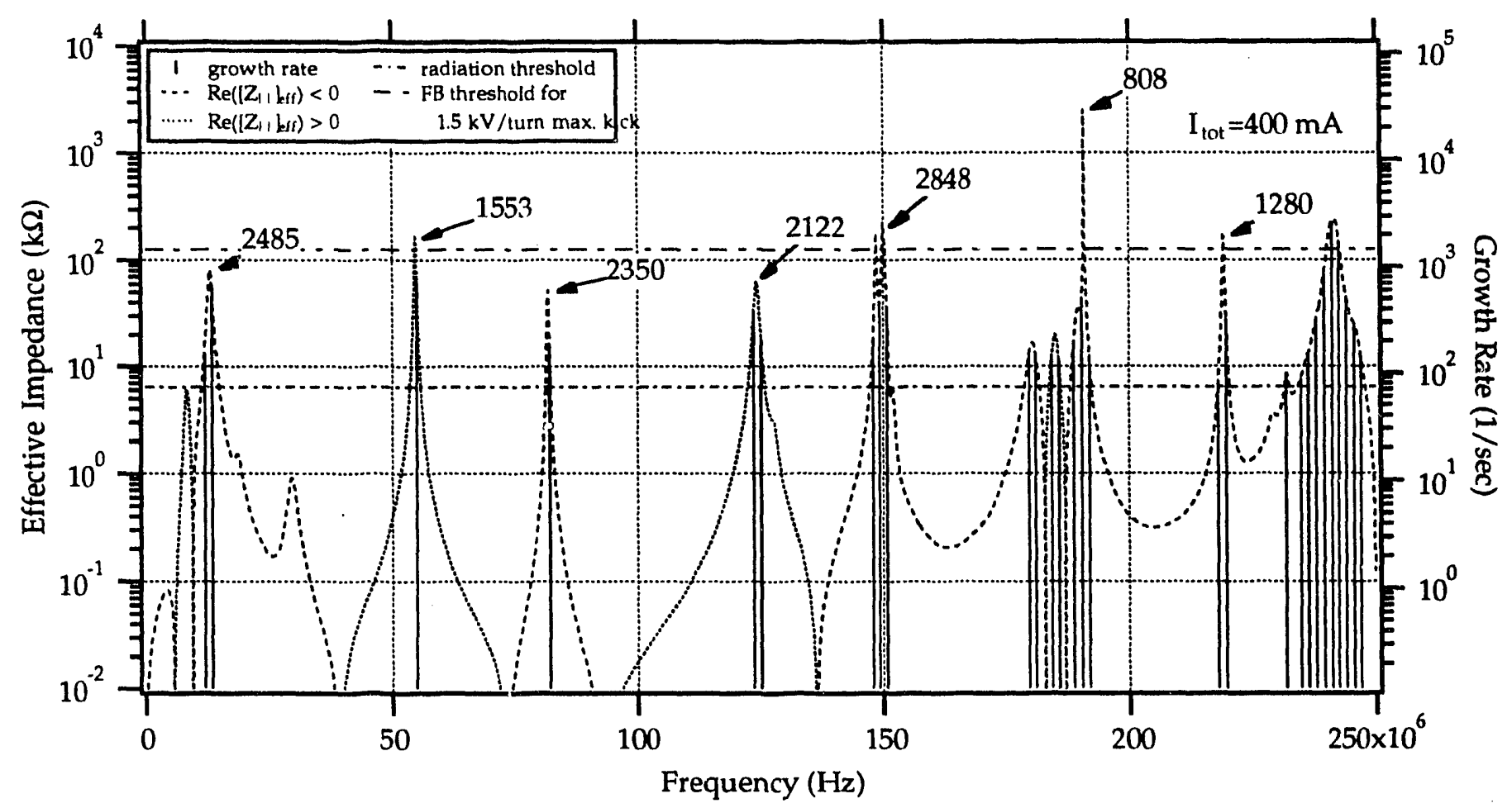

Figure 1: Longitudinal coupled-bunch growth rates and the effective impedance in the 0-250 MHz frequency band. The largest HOMs have been labeled according to their resonant frequency in $\mathrm{MHz}$. Only $\mathrm{CB}$ modes with grwoth rates greater than radiation damping are shown.

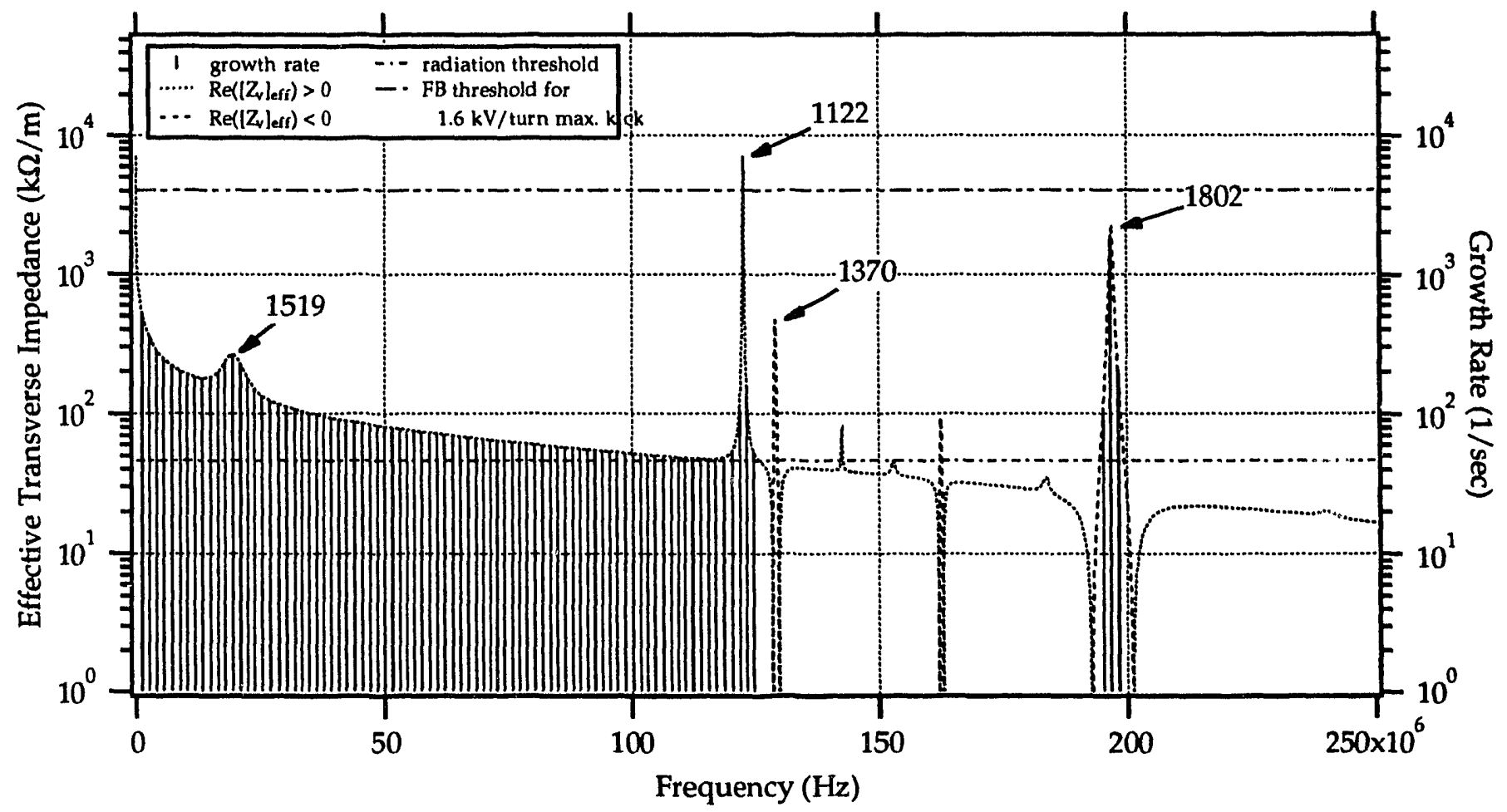

Figure 2: Vertical coupled-bunch growth rates and the effective impedance in the $0-250 \mathrm{MHz}$ frequency band from the $\mathrm{RF}$ cavity dipole HOM and the resistive wall impedance. 

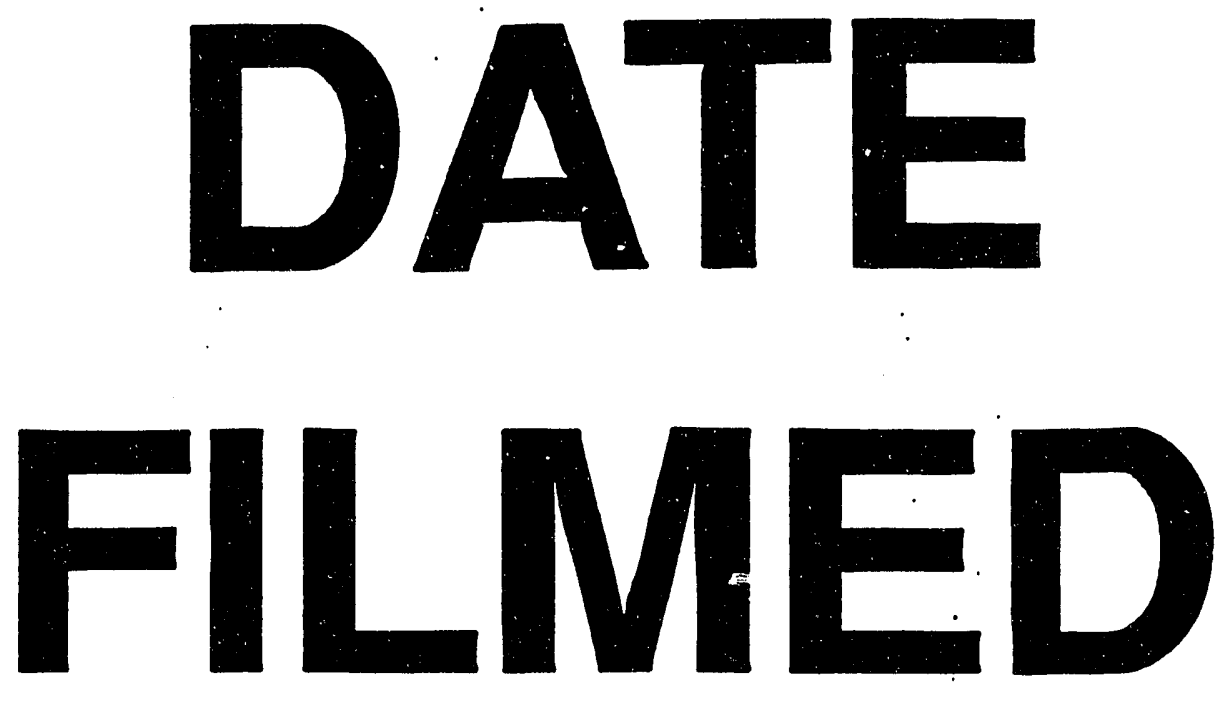

$10 / 19 / 93$
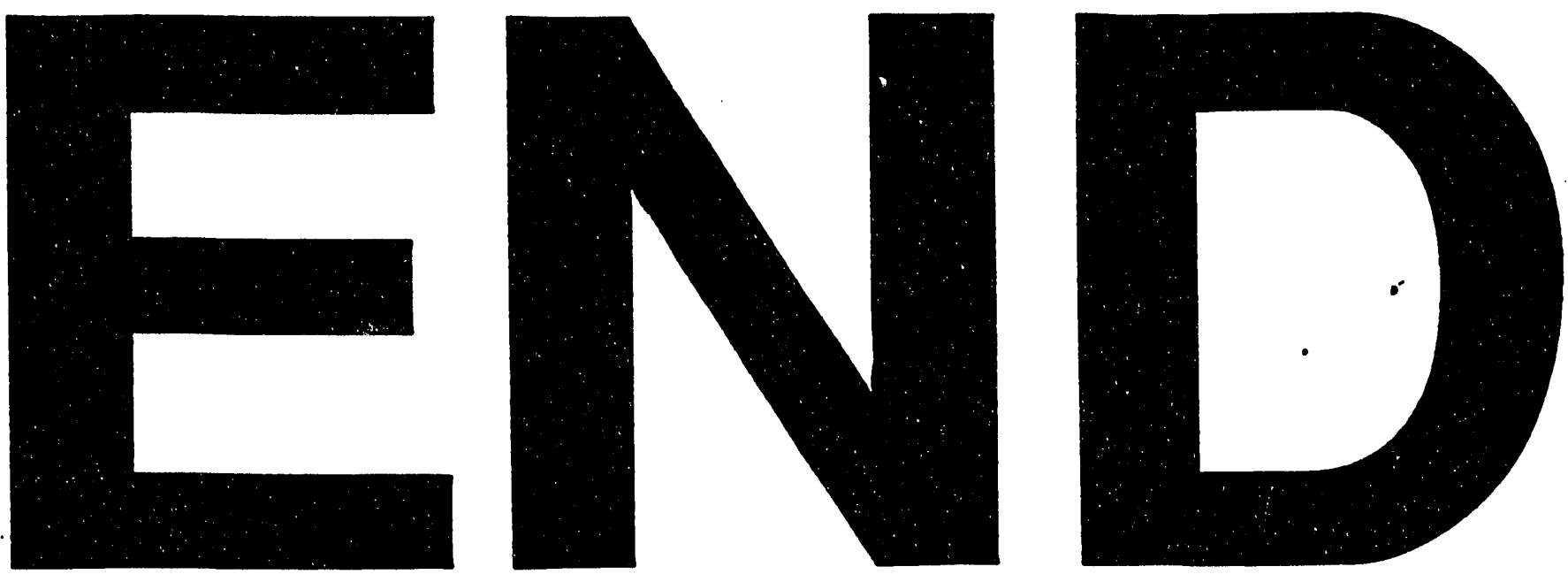
